\title{
ON DESCARTES' RULE OF SIGNS FOR MATRIX POLYNOMIALS
}

\author{
ThOMAS R. CAMERON AND PANAYIOTIS J. PSARRAKOS
}

Abstract. We present a generalized Descartes' rule of signs for self-adjoint matrix polynomials whose coefficients are either positive or negative definite, or null. In particular, we conjecture that the number of real positive (negative) eigenvalues of a matrix polynomial is bounded above by the product of the size of the matrix coefficients and the number of definite sign alternations (permanences) between consecutive coefficients. Our main result shows that this generalization holds under the additional assumption that the matrix polynomial is hyperbolic. In addition, we prove individual cases where the matrix polynomial is diagonalizable by congruence, or of degree three or less. The full proof of our conjecture is an open problem; we discuss analytic and algebraic approaches for solving this problem and ultimately, what makes this open problem non-trivial. Finally, we prove generalizations of two famous extensions of Descartes' rule: If all eigenvalues are real then the bounds in Descartes' rule are sharp and the number of real positive and negative eigenvalues have the same parity as the associated bounds in Descartes' rule.

Mathematics subject classification (2010): 15B57, 47B65, 47H60, 47J10.

Keywords and phrases: Descartes' rule of signs, matrix polynomials, eigenvalues, Hermitian matrices, definite matrices.

\section{REFERENCES}

[1] Al-Ammari, M. And Tisseur, F., Hermitian matrix polynomials with real eigenvalues of definite type. Part I: Classification, Linear Algebra Appl. 436, 10 (2012), 3954-3973.

[2] Anderson, B., Jackson, J. And Sitharam, M., Descartes' rule of signs revisited, Amer. Math. Monthly 105, 5 (1998), 447-451.

[3] Bensimhoun, M., Historical account and ultra-simple proofs of Descartes' rule of signs, De Gua, Fourier and Budan's rules, arXiv:1309.6664v5 [math.HO], 2016.

[4] Bini, D. A., Noferini, V. And Sharify, M., Locating the eigenvalues of matrix polynomials, SIAM J. Matrix Anal. Appl. 34, 4 (2013), 1708-1727.

[5] Bini, D. A. AND NofERIni, V., Solving polynomial eigenvalue problems by means of the EhrlichAberth method, Linear Algebra Appl. 439, 4 (2013), 1130-1149.

[6] CAmeron, T. R., Spectral bounds for matrix polynomials with unitary coefficients, Electron. J. Linear Algebra 30, (2015), 585-591.

[7] CuRTiss, D. R., Recent extensions of Descartes' rule of signs, Ann. of Math., (1918), 251-278.

[8] Descartes, R., La géometrie (Discours de la méthode, third part), Ed. of Leyde, 1637.

[9] Duffin, R. J., A minmax theory for overdamped networks, J. Rat. Mech. Anal. 4, (1955), $221-233$.

[10] Frazer, R. A., Duncan, W. J. And Collar, A. R., Elementary matrices, 2nd ed., Cambridge Univ. Press, London and New York, 1955.

[11] Gohberg, I., Goldberg, S. And KaAshoek, M., Classes of Linear Operators, vol. I, Birkhäuser Verlag, Basel, Switzerland, 1990.

[12] Gohberg, I., Lancaster, P. And Rodman, L., Matrix Polynomials, SIAM, Philadelphia, PA, 2009.

[13] Horn, R. A. And Johnson, C. R., Topics in Matrix Analysis, Cambridge University Press, New York, NY, 1991.

[14] LAnCASter, P., Lambda-matrices and Vibrating Systems, Pergamon Press, Oxford, United Kingdom, 1966. 
[15] Li, C.-K. And Rodman, L., Numerical range of matrix polynomials, SIAM J. Matrix Anal. Appl. 15, 4 (1994), 1256-1265.

[16] MARKuS, A. S., Introduction to the Spectral Theory of Polynomial Operator Pencils, AMS, Providence, RI, 1988.

[17] Melman, A., Generalization and variations of Pellet's theorem for matrix polynomials, Linear Algebra Appl. 439, 5 (2013), 1550-1567.

[18] Noferini, V., Sharify, M. AND TisseUR, F., Tropical roots as approximations to eigenvalues of matrix polynomials, SIAM J. Matrix Anal. Appl. 36, 1 (2015), 138-157.

[19] Tisseur, F. AND MeERbergen, K., The quadratic eigenvalue problem, SIAM Review 43, 2 (2001), 235-286.

[20] WANG, X. A simple proof of Descartes' rule of signs, Amer. Math. Monthly 111, 6 (2004), 525-526. 